\title{
Noninvasive detection of nasopharyngeal carcinoma based on saliva proteins using surface-enhanced Raman spectroscopy
}

Xueliang Lin

Duo Lin

Xiaosong Ge

Sufang Qiu

Shangyuan Feng

Rong Chen 


\title{
Noninvasive detection of nasopharyngeal carcinoma based on saliva proteins using surface-enhanced Raman spectroscopy
}

\author{
Xueliang Lin, ${ }^{\mathrm{a}}$ Duo Lin, ${ }^{\mathrm{a}, \mathrm{b}, *}$ Xiaosong Ge, ${ }^{\mathrm{a}}$ Sufang Qiu, ${ }^{\mathrm{c}, *}$ Shangyuan Feng, ${ }^{\mathrm{a}}$ and Rong Chen ${ }^{\mathrm{a}}$ \\ ${ }^{a}$ Fujian Normal University, Ministry of Education, Key Laboratory of Optoelectronic Science and Technology for Medicine, Fujian Provincial Key \\ Laboratory for Photonics Technology, Fuzhou, China \\ ${ }^{\mathrm{b}}$ Fujian University of Traditional Chinese Medicine, College of Integrated Traditional Chinese and Western Medicine, Fuzhou, China \\ ${ }^{c} F u j i a n$ Medical University Cancer Hospital, Fujian Provincial Key Laboratory of Translational Cancer Medicine, Department of Radiation Oncology, \\ Fuzhou, China
}

\begin{abstract}
The present study evaluated the capability of saliva analysis combining membrane protein purification with surface-enhanced Raman spectroscopy (SERS) for noninvasive detection of nasopharyngeal carcinoma (NPC). A rapid and convenient protein purification method based on cellulose acetate membrane was developed. A total of 659 high-quality SERS spectra were acquired from purified proteins extracted from the saliva samples of 170 patients with pathologically confirmed NPC and 71 healthy volunteers. Spectral analysis of those saliva protein SERS spectra revealed specific changes in some biochemical compositions, which were possibly associated with NPC transformation. Furthermore, principal component analysis combined with linear discriminant analysis (PCA-LDA) was utilized to analyze and classify the saliva protein SERS spectra from NPC and healthy subjects. Diagnostic sensitivity of $70.7 \%$, specificity of $70.3 \%$, and diagnostic accuracy of $70.5 \%$ could be achieved by PCA-LDA for NPC identification. These results show that this assay based on saliva protein SERS analysis holds promising potential for developing a rapid, noninvasive, and convenient clinical tool for NPC screening. ๑ 2017 Society of Photo-Optical Instrumentation Engineers (SPIE) [DOI: 10.1117/1.JBO.22.10.105004]
\end{abstract}

Keywords: surface-enhanced Raman spectroscopy; nasopharyngeal carcinoma detection; saliva protein; principal component analysis combined with linear discriminant analysis.

Paper 170416RR received Jun. 27, 2017; accepted for publication Sep. 21, 2017; published online Oct. 13, 2017.

\section{Introduction}

According to Global Cancer Statistics, cancer is one of the most common causes of death, with about 14.1 million people being diagnosed and 8.2 million people dying of cancer in 2012 worldwide. ${ }^{1}$ Nasopharyngeal carcinoma (NPC), a head and neck malignancy, is an endemic disease with high mortality rates in certain regions of southeastern Asia, including Hong Kong, Guangdong province of China, Fujian province of China, and some other South Asian countries. ${ }^{2-5}$ The five-year overall survival rate is about $90 \%$ for stage I NPC patients; however, the stage IV group has a poor five-year overall survival rate of $30.3 \%{ }^{6,7}$ Hence, early screening and effective treatment are essential for preventing the advancement of NPC and improving the five-year overall survival rate. Unfortunately, early detection remains a great challenge due to the insidious nature of NPC and the relative anatomical inaccessibility of the nasopharynx. At present, the common detection and screening methods for NPC mainly include electronic nasopharyngoscope examination, nasopharyngeal and neck magnetic resonance imaging, body positron emission tomography, bone emission CT, and biopsy, which suffer the disadvantages of being time-consuming, highcost, complex procedures, and based on subjective judgment of clinicians with varying levels of experience. ${ }^{8-10}$ Therefore, development of an alternative diagnostic method is of significant clinical value for NPC screening.

*Address all correspondence to: Duo Lin, E-mail: linduo1986@163.com; Sufang Qiu, E-mail: sfqiu@126.com
Raman spectroscopy (RS) is a powerful optical analytical tool with many advantages over conventional optical detection techniques. For example, RS has low water interference, high signal-to-noise ratio, and high sensitivity compared with Fourier infrared spectroscopy. ${ }^{11}$ Additionally, RS has a narrow peak width and multiplexing detection ability, leading to better analytical efficiency than fluorescence spectroscopy. ${ }^{12,13}$ RS has attracted significant attentions for its material analysis and biomedical applications, especially for cancer diagnosis, due to its ability to provide structural- and biochemical-specific information of macromolecules, such as proteins, nucleic acids, lipids, and so on. ${ }^{14,15}$ However, the conventional RS technique has some deficiencies, including its inherent weak Raman scattering efficiency and strong fluorescence background. For instance, the Raman signal of a biological molecule is $1 / 10,000$ of its fluorescence signal. To overcome these problems, surface-enhanced Raman spectroscopy (SERS) technology exploits the interaction between the biomolecules and metal nanoparticles (NPs) surface to dramatically increase the Raman signal (enhancement factors of up to $10^{13}$ to $10^{15}$ ), providing a more powerful and sensitive detection approach for cancer detection. ${ }^{2,16,17}$ Currently, SERS technology has been widely used to detect various cancers in human samples, such as DNA, RNA, cell, blood, and tissue. ${ }^{18}$ Compared with traditional polymerase chain reactions or immunoassays, biomarker detection based on SERS avoids use of expensive reagents and complex sample preparation steps. In

$1083-3668 / 2017 / \$ 25.00$ ๑ 2017 SPIE 
addition, SERS has many advantages over traditional fluorescent method, such as better multiplex capability and less photobleaching. ${ }^{19,20}$

Human saliva, as a body fluid, contains a large number of serum components, abundant protein, and metabolites, which may change when associated with neoplastic transformation. ${ }^{21}$ Comparing the above-mentioned human samples, saliva is regarded as an ideal medium for cancer diagnostics due to its rapid, noninvasive, and convenient collection procedure. ${ }^{22-24}$ Recently, application of SERS in saliva analysis was investigated extensively to detect several types of cancer. ${ }^{19,25-27}$ These preliminary results demonstrated the potential of the saliva SERS method for cancer detection.

To further verify the efficiency of saliva SERS in NPC detection, in this work, a large number of NPC saliva samples were collected, and a saliva sample analysis based on membrane protein purification was developed for saliva SERS detection. Moreover, principal component analysis combined with a linear discriminant analysis (PCA-LDA) diagnostic algorithm was employed to classify the saliva protein SERS spectra from the NPC group and the healthy group.

\section{Materials and Methods}

\subsection{Preparation of Human Saliva Samples}

In this study, the saliva samples were collected from 170 NPC patients with confirmed clinical and histopathological diagnosis of NPC lesions and 71 healthy volunteers. Detailed information on these samples is presented in Table 1. This study obtained ethical approval from Fujian Provincial Cancer Hospital (Fuzhou, Fujian, China). The collection process of the saliva samples is as follows: (1) after $12 \mathrm{~h}$ of overnight fasting, $1.5 \mathrm{ml}$ of saliva was collected from the study subjects who washed their mouth with water three times between 7 and 8 am; (2) the collected saliva was centrifuged at $13,900 \mathrm{rpm}$ for $10 \mathrm{~min}$ to remove oral epithelial cells and any residual food debris; and (3) the pure saliva samples were frozen at $-80^{\circ} \mathrm{C}$ until used.

Table 1 Clinical diagnosis of NPC patients and healthy subjects.

\begin{tabular}{lcc} 
Characteristic & NPC patients & Healthy volunteers \\
\hline Total number & 170 & 71 \\
Gender & & \\
Male & 106 & 41 \\
Female & 64 & 30 \\
Age (years) & & \\
$\leq 50$ & 92 & 36 \\
$>50$ & 78 & 35 \\
Clinical stage of NPC & & \\
I and II & 39 & NA \\
IV and III & 131 & NA \\
\hline
\end{tabular}

Note: NPC, nasopharyngeal carcinoma and NA, not applicable.

\subsection{Preparation of Silver Colloids}

Silver (Ag) colloids were synthesized using hydroxylamine hydrochloride and $\mathrm{Ag}$ nitrate according to the deoxidizing method reported by $\mathrm{Li}$ et al ${ }^{28}$ At first, $4.5 \mathrm{ml}$ sodium hydroxide solution was mixed with $5 \mathrm{ml}$ of $0.06 \mathrm{M}$ hydroxylamine hydrochloride solution. After that, the mixture was added rapidly to $90 \mathrm{ml}$ of $0.0011 \mathrm{M}$ silver nitrate aqueous solution. The resulting mixture was stirred to obtain uniformly gray $\mathrm{Ag}$ colloids. Meanwhile, the maximum absorption peak of Ag colloids was $418 \mathrm{~nm}$, and the Ag colloids NPs sizes were represented with a mean diameter of $35 \mathrm{~nm}$ by transmission electron microscopy (TEM) [Fig. 1(a)]. Finally, the final Ag colloids were obtained by centrifuging the solution at $10,000 \mathrm{rpm}$ for $10 \mathrm{~min}$ and removing the supernatant as the SERS substrate.

\subsection{Saliva Protein Purification and Surface- Enhanced Raman Spectroscopy Detection}

Figure 1(b) shows the schematic diagram of saliva protein purification and SERS detection. In brief, $10 \mu \mathrm{l}$ of the purified saliva sample was blotted onto the cellulose acetate (CA) membrane $(2 \times 8 \mathrm{~cm})$ by a trace pipette gun two times. In total, $20 \mu \mathrm{L}$ of each purified saliva sample was used. After the saliva sample was completely absorbed for about $5 \mathrm{~min}$, the membrane was washed for $5 \mathrm{~min}$ in $400 \mathrm{ml}$ of a special solution made up of $180 \mathrm{ml}$ of $95 \%$ ethanol, $200 \mathrm{ml}$ of distilled water, and $20 \mathrm{ml}$ of glacial acetic acid. This step aimed to remove any other components contained in the saliva sample and leave only the saliva proteins in the CA membrane. Then, the membrane was patted dry with a filter paper for about $10 \mathrm{~min}$. After that, the position of the CA membrane that only contained saliva proteins was cut into pieces and collected in a tube. Next, $150 \mu 1$ of acetic acid was added into the tube to dissolve the membrane fragments into a transparent gel. Then, $150 \mu \mathrm{l}$ of a prepared Ag colloids solution was added into the tube and the tube was placed into $37^{\circ} \mathrm{C}$ water for $20 \mathrm{~min}$. Finally, $2 \mu \mathrm{l}$ of liquid supernatant (protein-Ag NP mixture) was dripped onto a clean aluminum plate (Guantai Metal, Hebei, China) for SERS measurements. All SERS spectra of the saliva protein were collected with a confocal Raman microspectrometer (Renishaw plc, Gloucestershire, UK) using a Peltier cooled charge-coupled device camera under a 785-nm diode laser (a maximum power output of $5 \mathrm{~mW}$ ) in the range of 600 to $1750 \mathrm{~cm}^{-1}$. All spectra were recorded with $50 \times$ objective, $2 \mathrm{~cm}^{-1}$ spectral resolution, and $10 \mathrm{~s}$ acquisition time. The software package WIRE 2.0 was used for raw spectral acquisition.

\subsection{Data Processing and Multivariate Statistical Analysis}

All the raw SERE spectra of saliva proteins contain Raman scattering, fluorescence background, and noise signals. To obtain better Raman spectra, a Vancouver Raman algorithm based on a fifth-order polynomial fitting method was used to remove fluorescence background and noise signals. ${ }^{29}$ Then, all background-subtracted SERS spectra were normalized to the integrated area under the curve in the range of 600 to $1750 \mathrm{~cm}^{-1}$ for a better comparison of SERS spectral shape in the analysis. This sophisticated and robust diagnostic model based on PCALDA was used for Raman spectral analysis in saliva detection. In this study, PCA was used to reduce complex data sets and select the principal components (PCs) that account for the maximal 


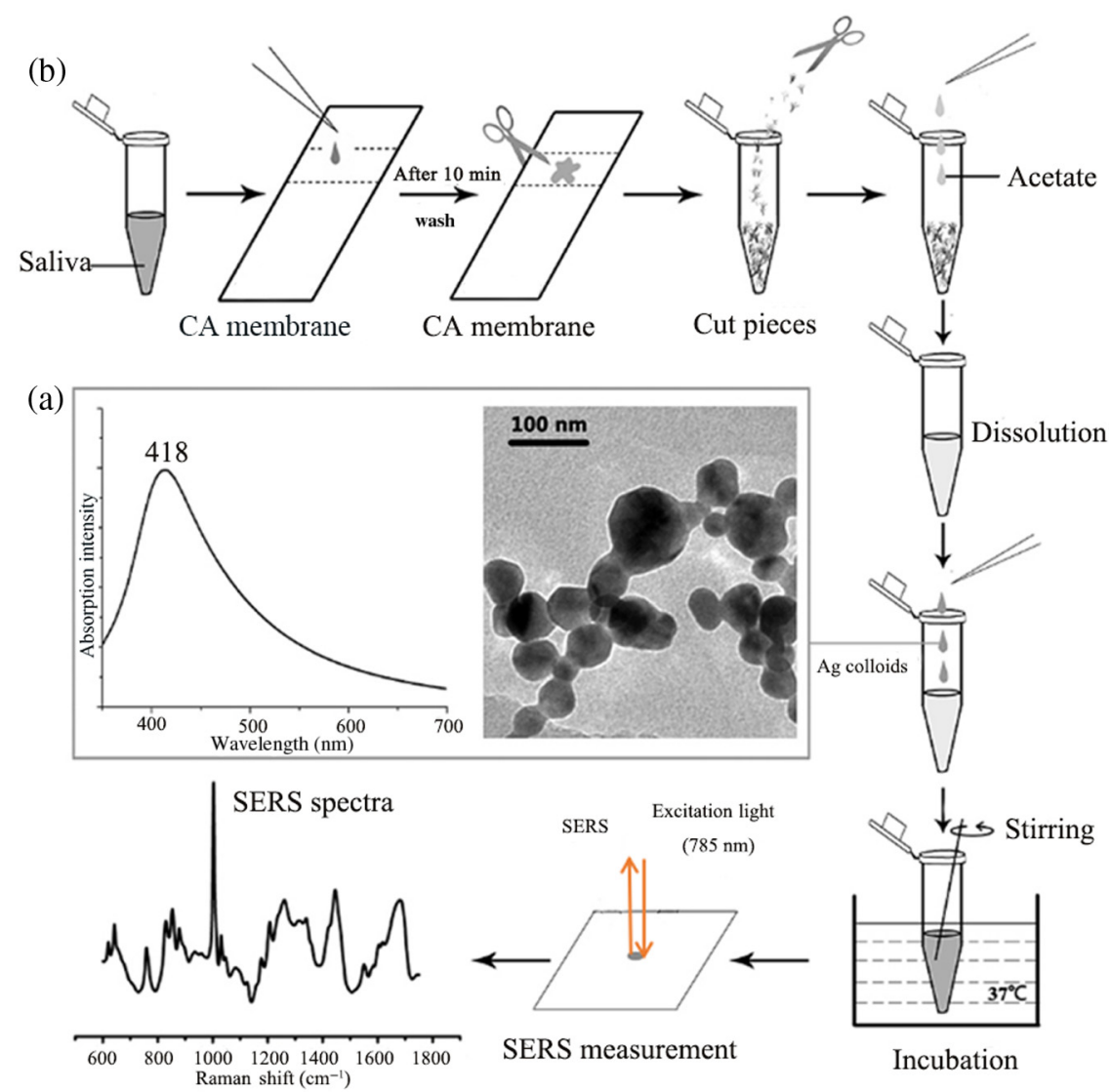

Fig. 1 (a) UV-visible-NIR absorption spectra of Ag colloids and TEM micrograph of the Ag colloidal surface and (b) the schematic diagram of saliva protein purification and SERS detection.

variances in the multidimensional data sets. ${ }^{30}$ To further analyze saliva protein SERS data, three diagnostically significant PCs $(p<0.05)$ were selected for one independent sample $t$-test. LDA was used to generate effective diagnostics using three diagnostically significant PCs with leave-one-out cross-validation methods. Receiver operating characteristic (ROC) curves were generated by successively changing the discrimination threshold levels to additionally evaluate the classifications of the multivariate statistical method for NPC diagnosis. ${ }^{30}$

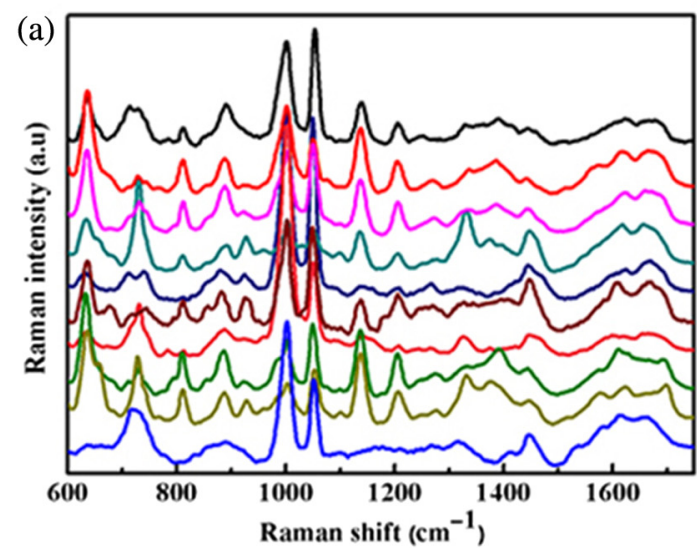

\section{Results and Discussions}

Figures 2(a) and 2(b) show the SERS spectra of untreated saliva samples of 10 patients with NPC and saliva protein samples after membrane protein purification from the same samples, respectively. Great variations in spectral features, including spectral intensity, position, and width, could be found in the untreated saliva SERS within the cancer group, making it difficult to achieve efficient diagnostics using SERS spectral analysis. This is explainable. A saliva sample contains a variety of native

Fig. 2 (a) Untreated saliva SERS spectra from 10 NPC saliva samples and (b) saliva protein SERS spectra using membrane protein purification method from the same samples. 

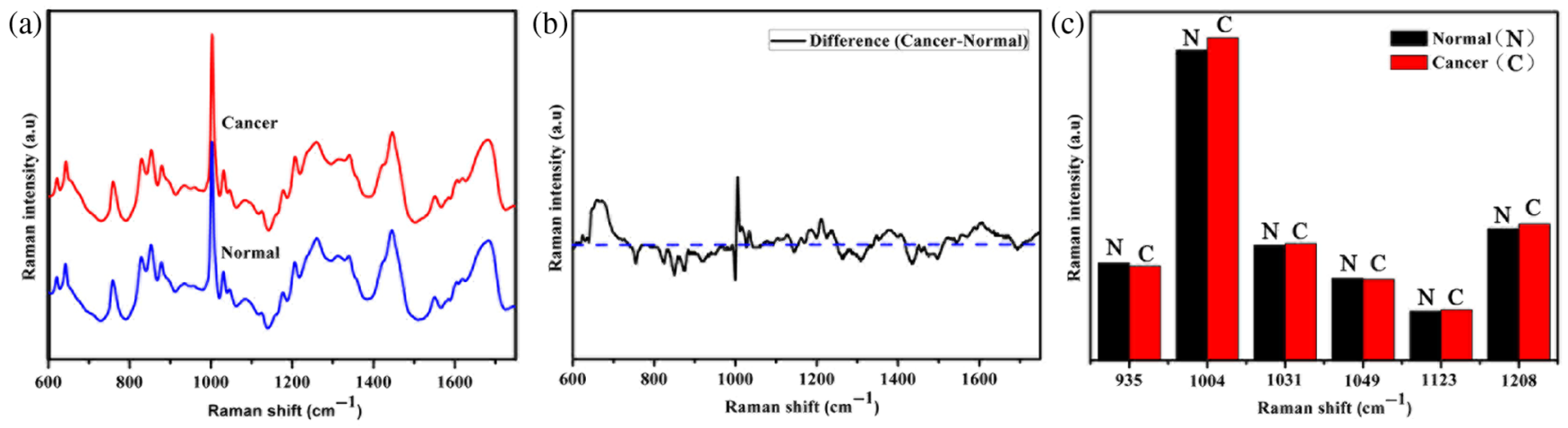

Fig. 3 (a) Comparison of the mean normalized SERS spectrum of purified proteins obtained from normal saliva (blue line, $n=71$ ) and cancer saliva (red line, $n=170$ ), (b) difference spectrum calculated from the mean SERS spectra between the NPC and normal groups, and (c) comparison of the mean intensities of the selected peaks between cancer (red pillar) and normal (black pillar) samples.

constituents (proteins, peptides, polynucleotides, and electrolytes) and exogenous substances (nonadherent oral bacteria, food remainders, traces of medications, or chemical products). ${ }^{31,32}$ Although preprocessing such as washing the mouth for saliva collection was implemented, there remained some exogenous substances in the saliva samples that generated prominent SERS signals. Thus, great SERS spectral variation from untreated saliva samples was probably attributed to the different exogenous substances among subjects. Interestingly, the reproducibility of SERS spectra [Fig. 2(b)] was dramatically improved by the saliva protein purification method developed. This method not only circumvented the limitations of raw saliva SERS detection but also provided a unique opportunity to use SERS to explore the changes in saliva proteins associated with cancer transformation.

Figure 3(a) shows the mean normalized SERS spectrum of purified whole proteins obtained from normal (blue line, $n=71$ ) and NPC (red line, $n=170$ ) saliva samples. The prominent SERS peaks located at around 621, 642, 760, 854, 878, 935, 959, 1004, 1031, 1049, 1123, 1175, 1208, 1265, $1337,1445,1552$, and $1684 \mathrm{~cm}^{-1}$ were clearly observed in both normal and NPC saliva protein samples. These prominent SERS peaks could be tentatively assigned to explain the changes in biological constituents in saliva protein samples as shown in Table $2 .{ }^{33-36}$ The strongest peaks at $760,1004,1265,1445$, and $1684 \mathrm{~cm}^{-1}$ existed in the measured saliva protein SERS spectra. Compared with the SERS spectra of NPC saliva proteins, the SERS spectra of normal saliva proteins had higher intensities at 621,935 , and $1049 \mathrm{~cm}^{-1}$ and lower intensities at 1004 , $1031,1208,1123$, and $1684 \mathrm{~cm}^{-1}$. The comparisons of saliva proteins SERS spectral intensities between the NPC and normal groups could be viewed more clearly in Figs. 3(b) and 3(c). These differences in spectral intensities demonstrated that saliva proteins SERS has a potential role for NPC detection. For instance, the SERS bands of phenylalanine (1004 and $1031 \mathrm{~cm}^{-1}$ ) were all related to the molecular stretching and bending mode of proteins, and they showed a lower SERS signal in the normal saliva protein samples than in the NPC saliva protein samples, indicating that an increase in the percentage of these proteins content in the total SERS-active in the NPC patients. Our group also observed that the content of phenylalanine was increased when associated with malignant transformation in cervical and NPC blood plasma by the SERS technique. $^{2,18}$ However, the intensity of the saliva protein
SERS spectrum exhibited decreased peaks at $935 \mathrm{~cm}^{-1}$ (praline and valine) and $1049 \mathrm{~cm}^{-1}$ (proteins) in NPC subjects, indicating that cancer patient saliva may be associated with a decreased concentration of these proteins. These results revealed that specific-protein changes between the NPC and normal groups could

Table 2 SERS peak positions and tentative vibrational mode assignments.

\begin{tabular}{lcc} 
Peak position $\left(\mathrm{cm}^{-1}\right)$ & Vibrational mode & Major assignments \\
\hline 621 & $\mathrm{C}-\mathrm{C}$ twisting mode & Phenylalanine \\
642 & $\nu(\mathrm{C}-\mathrm{S})$ & Tyrosine \\
760 & Ring breathing mode & Typtophan \\
854 & Ring breathing mode & Tyrosine \\
878 & $\nu(\mathrm{C}-\mathrm{C})$ & Hydro-oxyproline \\
935 & $\nu(\mathrm{C}-\mathrm{C})$ & Proline \\
959 & $\nu(\mathrm{C}-\mathrm{C})$ & $\alpha$-Helix, proline, \\
& & valine \\
1004 & $\nu(\mathrm{C}-\mathrm{C})$ & Phenylalanine \\
1031 & $\delta(\mathrm{C}-\mathrm{H})$ & Phenylalanine \\
1049 & $\nu(\mathrm{C}-\mathrm{O}), \nu(\mathrm{C}-\mathrm{N})$ & Proteins \\
1123 & $\nu(\mathrm{C}-\mathrm{N})$ & Proteins \\
1175 & $\delta(\mathrm{C}-\mathrm{H})$ & Tyrosine \\
1208 & $\nu\left(\mathrm{C}-\mathrm{C}_{6} \mathrm{H}_{5}\right)$ & Typtophan \\
1265 & $\nu(\mathrm{CN}), \delta(\mathrm{NH})$ & Amide III, collagen \\
1337 & $\mathrm{CH}_{3} \mathrm{CH} \mathrm{H}_{2}$ wagging & Collagen \\
1445 & $\delta(\mathrm{C}-\mathrm{H})$ & Collagen, lipids \\
1552 & $\nu(\mathrm{C}=\mathrm{C})$ & Typtophan \\
1684 & $\nu(\mathrm{C}=\mathrm{C})$ & Amide I \\
\hline & & \\
& &
\end{tabular}

Note: $\nu$, stretching vibration; $\delta$, bending vibration; and $\nu_{s}$, symmetric stretch. 

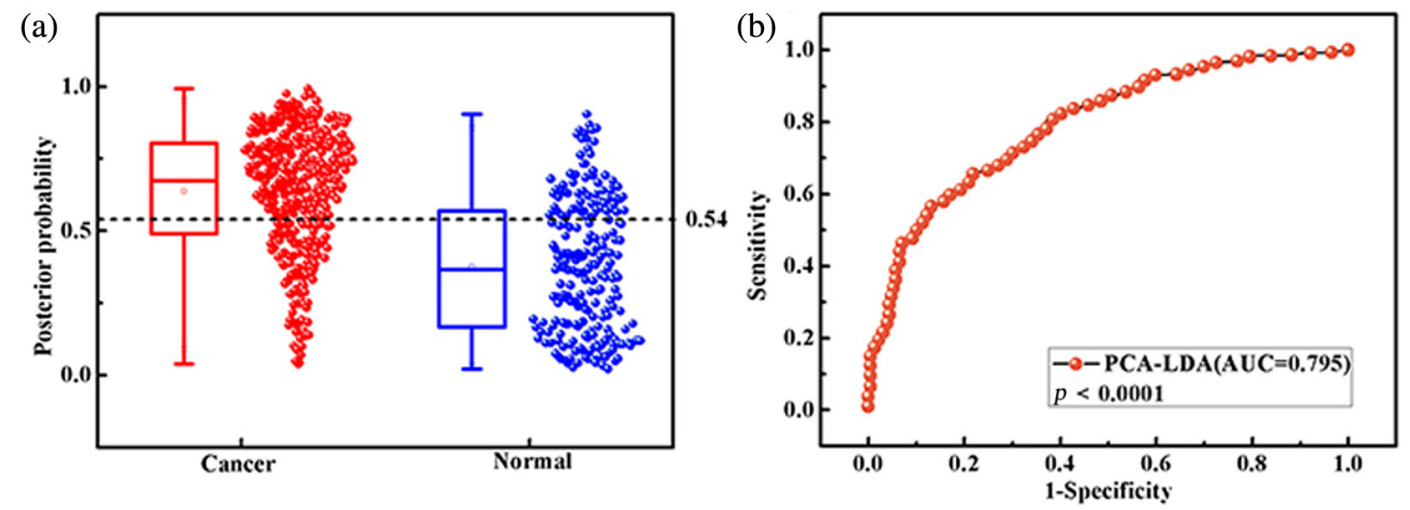

Fig. 4 (a) Scatter plots of the posterior probability of NPC (red triangles) and normal (blue circles) saliva samples using the PCA-LDA algorithm. The separation threshold was 0.54. (b) The ROC curve of discrimination results for saliva protein SERS spectra. The integration area under the ROC curve was 0.795.

be detected by SERS, suggesting promising potential of saliva protein SERS for NPC screening.

It should be noted that the simplistic peak intensities analysis above only uses limited information of SERS peaks, and more valuable diagnostic information contained in the SERS spectra has not been employed for spectral classification. Therefore, the PCA-LDA diagnostic algorithm was used to improve the diagnostic efficiency of the SERS technique by analyzing and classifying the saliva protein SERS spectra from NPC and normal subjects.

This sophisticated and robust diagnostic model based on PCA-LDA has been widely used for Raman spectral analysis in tissue, cell, saliva, and blood detection..$^{27,37,38}$ In this study, PCA was used first to reduce intensity variables within the raw Raman spectrum of saliva protein into a few PCs. Then, three PCs (PC5, PC6, and PC7) were selected to be the most diagnostically significant $(p<0.0001)$ as defined for discriminating normal and NPC groups by an independent sample $t$-test.

To further analyze saliva protein SERS data, all three diagnostically significant PCs were loaded into the LDA with the leave-one-out cross-validation method for generating an effective diagnostic model for saliva protein sample classification. Figure 4(a) shows the posterior probability plot of the healthy group (circles) and NPC group (triangles) based on the results of the PCA-LDA diagnostic model. As we can see, there are some plots overlapping in this figure. A discrimination threshold of 0.54 was employed for accurate discriminate between the healthy volunteers and NPC patients, yielding the diagnostic sensitivity, specificity, and accuracy of $70.7 \%$ (304/430), $70.3 \%(161 / 229)$, and $70.5 \%$ (465/659), respectively. To further evaluate the performance of saliva protein SERS for NPC diagnosis, an ROC curve was generated from the PCA-LDA data as shown in Fig. 4(b). The integration area under the ROC curve was 0.795 . These results further indicated that saliva protein SERS technology combining the PCA-LDA diagnostic algorithm has the potential as a rapid and noninvasive diagnosis method for NPC detection.

\section{Conclusions}

A rapid and convenient saliva SERS analysis method was developed for NPC detection. Using the membrane protein purification method, high-quality and reproducibility SERS spectra of saliva proteins were obtained, making it possible to reveal specific changes in proteins associated with cancer transformation. Furthermore, a diagnostic sensitivity of $70.7 \%$ and specificity of $70.3 \%$ could be achieved by the PCA-LDA diagnostic algorithm for NPC identification, demonstrating promising potential of this saliva SERS analysis method to be a noninvasive body fluid test for clinical NPC screening.

\section{Disclosures}

The authors declare that there are no conflicts of interest related to this article.

\section{Acknowledgments}

This work was supported by the National Natural Science Foundation of China (Nos. U1605253, 61210016, 61405036, and 61575043), the Innovation Team Development Plan by the Ministry of Education of China (No. IRT15R10), the National Natural Science Foundation of Fujian, China (Nos. 2017J01499 and 2015J01436), the Key Clinical Specialty Discipline Construction Program of Fujian (China), and the National Clinical Key Specialty Construction Program.

\section{References}

1. L. A. Torre et al., "Global cancer statistics, 2012," CA-Cancer J. Clin. 65(2), 87-108 (2015).

2. S. Feng et al., "Nasopharyngeal cancer detection based on blood plasma surface-enhanced Raman spectroscopy and multivariate analysis," Biosens. Bioelectron. 25(11), 2414-2419 (2010).

3. J. Ferlay et al., "Cancer incidence and mortality worldwide: sources, methods and major patterns in GLOBOCAN 2012," Int. J. Cancer 136(5), E359-E386 (2015).

4. M. C. Yu and J.-M. Yuan, "Epidemiology of nasopharyngeal carcinoma," Semin. Cancer Biol. 12, 421-429 (2002).

5. L.-L. Tang et al., "Global trends in incidence and mortality of nasopharyngeal carcinoma," Cancer Lett. 374(1), 22-30 (2016).

6. X. Sun et al., "Long-term outcomes of intensity-modulated radiotherapy for 868 patients with nasopharyngeal carcinoma: an analysis of survival and treatment toxicities," Radiother. Oncol. 110(3), 398-403 (2014).

7. Y. Chen et al., "Progress report of a randomized trial comparing longterm survival and late toxicity of concurrent chemoradiotherapy with adjuvant chemotherapy versus radiotherapy alone in patients with stage III to IVB nasopharyngeal carcinoma from endemic regions of China," Cancer 119(12), 2230-2238 (2013).

8. A. King et al., "The impact of 18F-FDG PET/CT on assessment of nasopharyngeal carcinoma at diagnosis," Br. J. Radiol. 81(964), 291-298 (2014). 
9. S.-X. Zhang et al., "Intravoxel incoherent motion MRI: emerging applications for nasopharyngeal carcinoma at the primary site," Eur. Radiol. 24(8), 1998-2004 (2014).

10. H. Kwok et al., "Genomic diversity of Epstein-Barr virus genomes isolated from primary nasopharyngeal carcinoma biopsy samples," J. Virol. 88(18), 10662-10672 (2014).

11. H. Kimura-Suda and T. Ito, "Bone quality characteristics obtained by Fourier transform infrared and Raman spectroscopic imaging," J. Oral Biosci. 591, 142-145 (2017).

12. S. Nie and S. R. Emory, "Probing single molecules and single nanoparticles by surface-enhanced Raman scattering," Science 275(5303), 1102-1106 (1997).

13. S. Schlücker, "Surface-enhanced Raman spectroscopy: concepts and chemical applications," Angew. Chem. Int. Ed. 53(19), 4756-4795 (2014).

14. S. Hong et al., "Live-cell stimulated Raman scattering imaging of alkyne-tagged biomolecules," Angew. Chem. Int. Ed. 53(23), 5827-5831 (2014).

15. K. Kong et al., "Raman spectroscopy for medical diagnostics-from in-vitro biofluid assays to in-vivo cancer detection," Adv. Drug Delivery Rev. 89, 121-134 (2015).

16. D. Lin et al., "Colorectal cancer detection by gold nanoparticle based surface-enhanced Raman spectroscopy of blood serum and statistical analysis," Opt. Express 19(14), 13565-13577 (2011).

17. J. Kneipp, H. Kneipp, and K. Kneipp, "SERS—a single-molecule and nanoscale tool for bioanalytics," Chem. Soc. Rev. 37(5), 1052-1060 (2008).

18. S. Feng et al., "Blood plasma surface-enhanced Raman spectroscopy for non-invasive optical detection of cervical cancer," Analyst 138(14), 3967-3974 (2013).

19. M. Vendrell et al., "Surface-enhanced Raman scattering in cancer detection and imaging," Trends Biotechnol. 31(4), 249-257 (2013).

20. K. C. Bantz et al., "Recent progress in SERS biosensing," Phys. Chem. Chem. Phys. 13(24), 11551-11567 (2011).

21. P. Dowling et al., "Analysis of the saliva proteome from patients with head and neck squamous cell carcinoma reveals differences in abundance levels of proteins associated with tumour progression and metastasis," J. Proteomics 71(2), 168-175 (2008).

22. M. Castagnola et al., "Potential applications of human saliva as diagnostic fluid," Acta Otorhinolaryngol. Ital. 31(6), 347-357 (2011).

23. K. Thomadaki et al., "Whole-saliva proteolysis and its impact on salivary diagnostics," J. Dent. Res. 90(11), 1325-1330 (2011).

24. L. R. Bigler et al., "Salivary biomarkers for the detection of malignant tumors that are remote from the oral cavity," Clin. Lab. Med. 29(1), 71-85 (2009).

25. K. W. Kho et al., "Surface enhanced Raman spectroscopic (SERS) study of saliva in the early detection of oral cancer," Proc. SPIE 5702, 84-91 (2005).

26. X. Li, T. Yang, and J. Lin, "Spectral analysis of human saliva for detection of lung cancer using surface-enhanced Raman spectroscopy," J. Biomed. Opt. 17(3), 037003 (2012).

27. S. Qiu et al., "Non-invasive detection of nasopharyngeal carcinoma using saliva surface-enhanced Raman spectroscopy," Oncol. Lett. 11(1), 884-890 (2016).

28. S.-M. Li et al., "Rapid microwave-assisted preparation and characterization of cellulose-silver nanocomposites," Carbohydr. Polym. 83(2), 422-429 (2011).

29. J. Zhao et al., "Automated autofluorescence background subtraction algorithm for biomedical Raman spectroscopy," Appl. Spectrosc. 61(11), 1225-1232 (2007).
30. U. Demšar et al., "Principal component analysis on spatial data: an overview," Ann. Assoc. Am. Geogr. 103(1), 106-128 (2013).

31. P. D. V. de Almeida et al., "Saliva composition and functions: a comprehensive review," J. Contemp. Dent. 9(3), 72-80 (2008).

32. S. Singh et al., "Saliva as a prediction tool for dental caries: an in vivo study," J. Oral Biol. Craniofac. Res. 5(2), 59-64 (2015).

33. L.-J. Xu et al., "Label-free detection of native proteins by surfaceenhanced Raman spectroscopy using iodide-modified nanoparticles," Anal. Chem. 86(4), 2238-2245 (2014).

34. D. Kurouski et al., "Amide I vibrational mode suppression in surface (SERS) and tip (TERS) enhanced Raman spectra of protein specimens," Analyst 138(6), 1665-1673 (2013).

35. Z. Huang et al., "Near-infrared Raman spectroscopy for optical diagnosis of lung cancer," Int. J. Cancer 107(6), 1047-1052 (2003).

36. S. Teh et al., "Diagnostic potential of near-infrared Raman spectroscopy in the stomach: differentiating dysplasia from normal tissue," $\mathrm{Br}$. J. Cancer 98(2), 457-465 (2008).

37. D. Lin et al., "Label-free blood plasma test based on surface-enhanced Raman scattering for tumor stages detection in nasopharyngeal cancer," Sci. Rep. 4, 4751 (2014).

38. Z. Li et al., "Raman microspectroscopy as a diagnostic tool to study single living nasopharyngeal carcinoma cell lines," Biochem. Cell Biol. 91(3), 182-186 (2013).

Xueliang Lin received his BS degree in physics from Longyan University in 2015. He is currently working at the Institute of Laser and Optoelectronics Technology and working toward his master's degree in optics at Fujian Normal University, China. His research focuses on the application of surfaced-enhanced Raman spectroscopy (SERS) in the diagnosis of nasopharyngeal carcinoma (NPC).

Duo Lin received his master's degree in physical electronics from Fujian Normal University in 2012. He is currently working at the Institute of Laser and Optoelectronics Technology and working toward his PhD in optics at Fujian Normal University, China. His research focuses on the application of SERS in biomedical diagnosis.

Xiaosong $\mathrm{Ge}$ received his BS degree communication engineering from Changzhou University in 2015. He is currently working at the Institute of Laser and Optoelectronics Technology and working toward his master's degree in physical electronics at Fujian Normal University, China. His research focuses on the application of SERS in biomedical diagnosis.

Sufang Qiu received her PhD from Fujian Medical University in 2016. Currently, she is a chief physician of Fujian Tumor Hospital, China. She is an oncologist and a master tutor. Her research interest focuses on radiation therapy and diagnosis of NPC.

Shangyuan Feng received his PhD from Fujian Normal University in 2011. He started a postdoctoral position at BC Cancer Agency, Canada, in 2013. Currently, he is an associate professor at the School of Optoelectronics and Information Engineering, Fujian Normal University, China. His research interest focuses on the application of SERS in biomedical diagnosis.

Rong Chen was the director of Key Laboratory of Optoelectronic Science and Technology for Medicine, Ministry of Education. Currently, he is a doctoral tutor at the School of Optoelectronics and Information Engineering, Fujian Normal University, China. His research interest is the application of Raman spectral to detect NPC. 